\title{
Context and Position in a Systemic Approach to Capacity Development
}

\author{
Carl Jackson
}

\begin{abstract}
This article discusses a process of facilitating capacity development in the Africa Adapt network using a systemic approach. The author reflects on the extent to which the context of Africa Adapt enabled a systemic approach, and how the practice of facilitating capacity development in this context differed from the theory on which it was based. The article argues that a systemic approach to facilitating capacity development has been enabled by the complicated context of Africa Adapt. However, the approach would have been strengthened by ensuring a more dynamic understanding of the system, and a more explicit acknowledgement of the positional power of capacity development facilitators within this context.
\end{abstract}

\section{Introduction}

Africa Adapt is a knowledge sharing network established by ENDA, FARA, ICPAC and IDS $^{1}$ as part of the IDRC and DFID funded Climate Change Adaptation in Africa programme. ${ }^{2}$ As one of two knowledge sharing capacity development advisers (the other being Catherine Fisher of IDS) I have collaborated with Africa Adapt for the last two and a half years. This article takes a step back from this emerging network of climate adaptation actors in Africa to reflect on the interplay between systems and context in facilitating capacity development. This article is intended to reflect on and draw out some lessons.

The process of facilitating capacity development in knowledge sharing is central to the Africa Adapt project. As advisers to Africa Adapt, our focus has been on four individuals, the Knowledge Sharing Officers (KSOs) - one based at each of four partner organisations co-hosting the network. KSOs are the primary actors in achieving the project ambitions of enhancing access to knowledge assets for climate adaptation. However, while our focus has been on the KSOs, we have tried not to see them in isolation. So, we have sought to take a systemic approach to facilitating capacity development, exploring the interactions between change at the organisational, sector/network and societal level as well as the individual. In saying this, we are thinking about a system with the capacity to share knowledge on climate adaptation as encompassing the people, processes, resources, preconditions and intentions that create the Africa Adapt network. In our approach, we have also sought to identify different kinds of capabilities - not only the capability to deliver particular outputs, but also the ability to relate, to adapt and to achieve coherence. Thus our facilitation of capacity development interventions looks not only at skills required to deliver on specific objectives, but the context in which those skills are applied, the ability of individuals and organisations to shape the environment in which they work, and the ways in which individuals, organisations and networks set and work towards objectives over time. In Africa Adapt, the facilitation of capacity development is seen as a process of learning and change, a process in which capacity development interventions are undertaken in order to stimulate change at different levels within the system.

The process of facilitating capacity development within Africa Adapt has involved more than half a dozen related interventions, and after two years' practice it seemed timely to ask:

To what extent has the context of Africa Adapt enabled a systemic approach to the

IDS Bulletin Volume 41 Number 3 May 2010 (C) 2010 The Author. Journal compilation (C) Institute of Development Studies Published by Blackwell Publishing Ltd, 9600 Garsington Road, Oxford OX4 2DQ, UK and 350 Main Street, Malden, MA 02148, USA 
facilitation of capacity development and for whom?

- How has the practice of facilitating capacity development in this context differed from our theoretical concepts and what accounts for it?

\section{Background}

The Climate Change Adaptation in Africa (CCAA) programme began in 2006. It aims to improve the capacity of African countries to adapt to climate change in ways that benefit the most vulnerable. It works to establish a selfsustained African body of expertise on adaptation that responds to needs defined by African communities, decision makers, and institutions' (IDRC 2007). Participatory action research is the largest part of the CCAA programme, along with communications and networking, education and training, and monitoring and evaluation. Africa Adapt is the main activity of the CCAA networking strand. Africa Adapt itself aims 'to facilitate the flow of climate change adaptation knowledge for sustainable livelihoods between researchers, policy makers, civil society organisations and communities who are vulnerable to climate variability and change across the continent' (Africa Adapt 2009). Four organisations came together to develop Africa Adapt in 2008. Environment and Development in the Third World (ENDA-TM), a major international NGO, based in Senegal, the Forum for Agricultural Research in Africa (FARA), a pan-African agricultural research organisation with its secretariat in Ghana, IGAD Climate Predictions and Applications Centre (ICPAC), a continentleading climate science organisation based in Kenya, and the Institute of Development Studies (IDS), a research organisation with considerable experience of developing and hosting knowledge services on international development issues, based in the UK. The Institute of Development Studies is the initial lead organisation in the partnerships, with leadership being transferred to one of the other partners during phase one.

\subsection{Knowledge sharing officers in Africa Adapt}

Experience suggests that building and sustaining a network requires dedicated individuals embedded within key strategic organisations. The first Africa Adapt project development meeting produced common elements of a job description for hiring KSOs and then moved to quickly appoint staff. From the outset, we
Figure 1 Five dimensions of capability

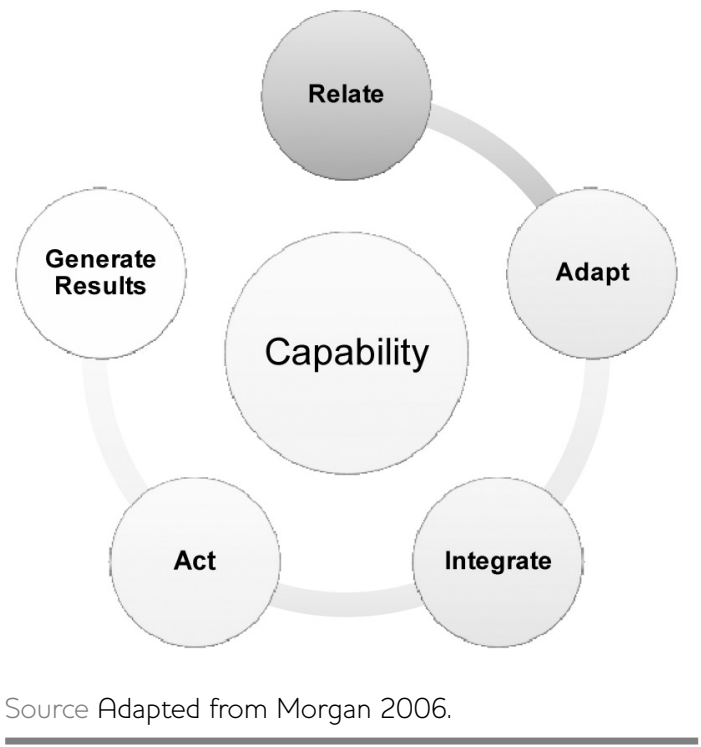

understood that the success of the KSOs would depend on the willingness of each partner to invest in the knowledge sharing capability of their organisation. This investment would ensure that the new KSO position was seen as a key part of each organisation's development and growth, and hence integrated within standard working practices and cultures. Accordingly, the KSOs were line managed by more senior members of their organisation who make up the Africa Adapt management team.

In conceiving of the new job descriptions, the role as knowledge intermediary and facilitator of the emerging Africa Adapt network was placed centre stage. As part of the KSO team, each KSO's responsibility was to facilitate knowledge sharing on climate through content management (e.g. using innovative and participatory methods to synthesise and share knowledge and persuading others to do the same); network management (e.g. matchmaking relationships, growing membership and supporting self-governance); engagement (e.g. facilitating face-to-face workshops and seeking feedback on the network); collaboration (e.g. developing relationships with participatory action research projects and other knowledge producers); and coordination activities (e.g. supporting the Africa Adapt project through good communication and stewardship of resources). While KSOs would likely be recruited with significant knowledge sharing and communications experience, they would also 
Figure 2 Self-assessment of confidence in areas of KSO responsibility

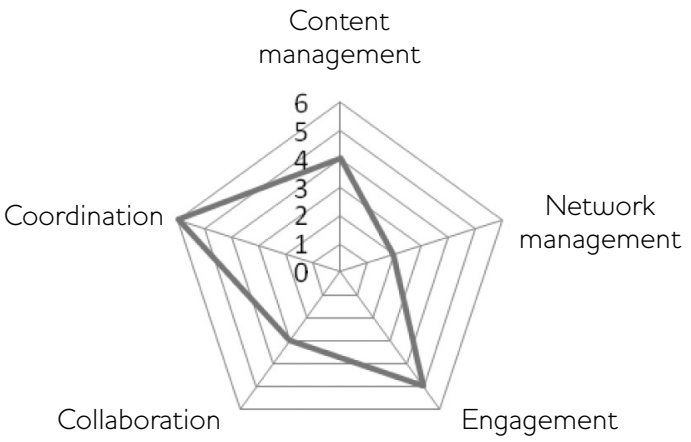

require support to develop their capabilities in light of the particular objectives of Africa Adapt as their new employing organisation.

\section{Facilitating capacity development for KSOs}

Africa Adapt's approach to facilitating capacity development is based on thinking from the Institute of Development Studies (Taylor and Clarke 2008) and from the European Centre for Development Policy Management (ECDPM). Peter Morgan of ECDPM defines capacity as 'the overall ability of a system to create value' (Morgan 2006: 8). Morgan highlights five areas where capabilities can be developed. These five capabilities are separate but interdependent (Figure 1). In designing the common training and individualised professional development plans for the KSOs, following Morgan, we intended that five main types of capability would be supported:

- Capability to do/deliver/act (functional capacity): This would be affected by KSOs' individual skills and abilities and the resources (physical, technical, financial, etc.) that are available to undertake activities;

- Capability to create/maintain space to generate results (operational capacity): How KSOs are able to create a working environment in which they can achieve objectives;

- Capability to relate (legitimacy in the eyes of stakeholders);

- Capability to innovate/adapt (innovative capacity): particular capacities can go out of date or become irrelevant not because of a change in the individual but a change in the external environment;
Capability to achieve coherence/integration (integrating structures inside the system).

\section{Systemic approach}

Our approach to facilitating capacity development sought to be systemic, in particular by being aware of the benefits of working at multiple and interconnected levels - namely individual, organisational, sectoral and societal. So, for instance, we assessed individual capability against skills and experience in the KSO job descriptions and recommended personal development plans. These plans were able to build on particular specialities and needs of individual KSOs. As KSOs had particular skill sets already, the personal development plans also included the objective of promoting peer support, sharing and skills transfer between each of the KSOs. At the next level, we assessed the organisational environment and existing level of internal capacity development support that would enable or constrain KSOs' work and recommended organisational support frameworks. At the sectoral level we sought to understand the KSO role as one among others in the information and knowledge intermediary world and sought to connect KSOs with their external peers' existing networks. Finally, at the societal level, we sought to assess the knowledge domain for climate adaptation within Africa (taking Kenya as an example) and recommended innovations that KSOs could explore with social partners to achieve the objectives of Africa Adapt.

Figure 2 is an example of one of the tools used to assess KSOs' capabilities. In this case, each KSO was asked to score their levels of confidence on assuming the role in key areas of future responsibility. A visual diagram was used to translate the scores into a composite visual measure of starting confidence. This was then used to facilitate discussion between the KSO and their mentor as to how capability in areas of lower confidence could be explored and built up during the first year of their professional development programme.

Our approach to facilitating capacity development also sought to be systemic by building in feedback loops for learning, by encouraging reflection by KSOs. To do this, the knowledge sharing capacity development advisers provided monthly mentoring sessions to 
each KSO. We also sought to respond to the holistic nature of opportunities and needs to develop capacity, by consciously using diverse interventions (e.g. a mix of methods, different lengths and cycles of engagement and a range of different people). Understanding capability systemically also meant a mix of hard and soft interventions. Hard, for example, being more focused on technical and procedural skills (e.g. skills in digital photo and video production and editorial procedures for publication) and soft focused on skills with a stronger empathic or creative element (e.g. facilitating relationships and exploring unfamiliar responsibilities through role play).

\section{The Africa Adapt context}

The context of Africa Adapt is a complicated one, as we will see below, but ultimately has many positive effects for a systemic approach to facilitating capacity development. The main dimensions to highlight are geography, language, sector focus, methodologies and sequencing. Africa Adapt's multiple partners are based in Senegal, Ghana, Kenya, UK and Canada, meaning that the project is a widely geographically distributed effort. This makes for more complicated project coordination in terms of divergent time zones, long-distance travel for face-to-face meetings and diverse ways of working. It is also a bilingual partnership with a formal commitment to working in French and English (and with other languages among core staff including Amharic, Wolof and Spanish). This entails time costs to ensure translation, as well as incurring potential costs from not doing so (e.g. partial understanding). The partner organisations are also active in diverse sectors including agriculture, energy, meteorology, and social policy - this diversity intentionally prefiguring the intended composition of the network. The relationships between these sectors are being re-framed through the topic of climate adaptation, but the nature of these relationships is not always self-evident. The very diverse methodologies and approaches each partner brings to its work is a reflection of their different sector backgrounds - including policy formulation and governance, social mobilisation and advocacy, statistical modelling and forecasting, action research and knowledge management. The jargon of methodology can obscure shared principles and assumptions, but fundamental differences did exist between approaches that required respect. The structure of the partnership for Africa Adapt is also complicated due to an in-built process to transfer project leadership within the first planning cycle through a competitive process and due to the rapidly changing structures and priorities of organisations working on a high profile issue like climate change.

\section{The extent to which context enables a systemic approach}

Taking a systemic approach to facilitating capacity development is, in many ways, enabled by the complicated context of Africa Adapt. But why? For one, the widely geographically distributed nature of the partnership means that decision-making power is multi-centred and uneven. In practice, coalitions of influence within the partnership shift in quite fluid ways. It certainly seems like the systemic approach of working at multiple and interconnected levels is more compatible in this context, than in one where power is more centralised and able to block a sometimes organisationally challenging intervention. The inherent flexibility of a systemic approach also means that the approach can be more agile in responding to changes in demand. Second, the inherent need for support for learning across the sectoral and methodological differences of the partner organisations means that meanings are more provisional and contestable. Because of this, there was already a momentum to look outside each organisation to KSO peers in partner organisations and to peers in other networks. This is highly compatible with a systemic approach that seeks to address capacity holistically rather than just focusing internally on individual organisations or staff.

The complicated context of Africa Adapt is less enabling of a systemic approach to facilitating capacity development in terms of the higher demand this placed on the knowledge sharing capacity development advisers to remain up to date with the changing properties of the system. In retrospect, it is clear that more effort should have been put into regularly refreshing our understanding of the system and iterating the implementation of the approach. In a less complicated context, taking a snap-shot of the system and relying on it for longer would have been less risky. 


\section{Who is enabled by the context?}

In terms of who is enabled by this complicated context for capacity development, three issues stand out: language, disciplinary focus and motivation. First, because of the centrality of English and French to Africa Adapt, those with the strongest bilingual skills in these two languages are more able to take advantage of opportunities to apply new capabilities acquired in other areas (e.g. media skills, research methods). Although all KSOs are bilingual, other language combinations were not as valuable in this context. Second, interdisciplinary thinkers seem to have been empowered because the complicated context put a premium on ability to respond to and interpret diverse drivers of change in the emerging knowledge domain of climate adaptation. Third, the complicated management structure of Africa Adapt, characterised by significant levels of delegation and multiple reporting lines favoured KSOs who were self-starters and happy to go for an entrepreneurial approach to applying new capabilities in delivering the project.

\section{Difference between practice and theory}

Comparing the practice of facilitating capacity development to our theory two and a half years on, three main divergences stand out. In theory, the knowledge sharing capacity development advisers' mapping of the knowledge domain for climate adaptation in Africa should have helped to put capability in its wider context by suggesting activities that KSOs could explore with social partners to achieve Africa Adapt's objectives. In practice, the knowledge domain map was little used because, in being comprehensive, it was too detailed with too many options for action at a time when KSOs were already busy with the launch of the Africa Adapt website. So the timing of this intervention was wrong. It may also have gone beyond the perceived mandate of capacity development.

The provision of individual mentoring for each KSO should, in theory, have provided a space outside of the line management relationship, within which KSOs were able to explore and address more complex or emergent capability needs. In practice the mentoring relationship only partly achieved this because, despite not being line managers, the mentors were staff based in the initial lead partner organisation (IDS) and colleagues of the IDS programme manager for Africa Adapt. As such, the mentoring space created could not be seen as genuinely neutral by the partners, and initiatives coming out of it were sometimes not given as much room to grow as might have been the case if the mentors were independent of all partners' agendas. On the plus side, mentoring did provide a chance to take a step back from day-to-day activities and reflect, with the regular sessions carving out time for reflection and learning, which is otherwise difficult to make. The other positive was that mentoring was very useful in providing ideas about different approaches to problem solving and making things seem more manageable for KSOs.

Our approach to facilitating capacity development was unrealistic, in as much as the theory of a systemic approach implied the ability to understand and influence all the factors affecting the KSOs' capability. Starting with this fallacy of complete control meant that in practice, this novel approach was only partly able to influence the organisational context for each $\mathrm{KSO}$, because the momentum of process and culture within each organisation had a wider and sustained purpose. In theory, our approach to facilitating capacity development had sought to understand all relevant factors influencing the KSOs' capability. In practice, we had failed to account for at least one very significant factor, namely the unique relationship that would emerge between $\mathrm{KSO}$ and line manager in each organisation. We had not established a method that would provide insight into the emerging relationship and instead relied on partial accounts from individuals. Neither did we have much influence over this relationship. We considered using project line management as a source of influence but this would have tended to undermine the consensual nature of our approach and so was not used. A systemic approach to facilitating capacity development may also not have been fully bought into by partners, in terms of accepting the changes it could ask of their organisations.

\section{Has the KSO peer group begun to co-create the context for a culture of knowledge sharing?}

One of the objectives for the process of facilitating capacity development was that fairly quickly, the KSOs would begin mentoring each other through creating their own peer group. As well as adding to the sustainability of each KSO's 
role and building resilience in the system, the expectation was that the KSO peer group would begin to co-create the context for meeting the wider objective of Africa Adapt to demonstrate the value of a culture of knowledge sharing to key audiences. What of progress here? It is true that co-mentoring between the KSOs began very early on. A peer-assist session was facilitated as part of the capacity development workshop that brought KSOs together for the first time. That practice, and others like it, such as bilateral Skype chats, task balancing and, most recently, exchanges between KSOs, have developed a functioning peer group. However, it is not yet the case that the KSO peer group is co-creating the context for the wider success of the project. There is some evidence that KSOs' use of collaborative and distributed working methods is requiring others within the partner organisations to orientate themselves towards KSOs' knowledge sharing culture. For example, the line managers' group expressed a collective unease with the spontaneous monthly Skype meetings that KSOs were holding and requested regular notes on the topics discussed. However, the influence has primarily promoted a reaction from existing ways of working (calls for integration with existing systems, compliance with traditional norms and recapture of innovation space), rather than seeding transformatory changes.

KSOs have carved out and, to date, sustained a space where a culture that values collaborative and distributed knowledge sharing can exist. They have done this primarily by working differently and - effectively; bringing to bear upon the challenges of Africa Adapt their own assumptions and skills in knowledge work. This is partly, but not entirely, a generational factor. The majority of KSOs are digital natives for who access to the internet, mobiles and open models of communication are regular and unremarkable parts of everyday life. But it is also because the essence of the roles they occupy, as facilitators and mediators of others' knowledge, has placed them in a sometimes ambiguous and pioneering role that sees them spanning between their own organisation and others in the emerging Africa Adapt network. The hybridity of the KSO role calls upon each individual to draw upon that which is plural within themselves if they are to successfully make sense of and negotiate the space to act upon the everyday challenges and innovate within opportunities to support Africa Adapt.
To the extent that the KSO peer group has not yet co-created the context for the wider success of the project, this is perhaps because there has been relatively little chance for face-to-face interaction between the KSO peer group, nor between the KSO peer group and the collective management group (so far only once a year). Also, there have been different levels of encouragement for KSOs to participate actively in peer networking. Consequently, full peer group activity has been limited to those more limited numbers of activities where interests spontaneously converged.

\section{Reflections}

The perceived partiality of our facilitation of the capacity development process has had a big impact on our ability to be advocates for harder choices in developing capacity, especially when new choices spring up in the course of a workshop or mentoring session, and there is no planned activity to justify the action to managers. At times like this, it is easy for the facilitator's relationship to wider power dynamics within the partnership of organisations to be called into question should anyone need to delay a hard choice. This suggests that the positionality of the facilitator of a capacity development process matters and needs to be as neutral as possible.

The model of mentoring the individual and then peer group took us a long way, but we may have missed a significant opportunity to cross-fertilise these sets by also having periodic collective reflections, bringing together all KSOs and mentors to calibrate progress and compare notes on challenges and opportunities. This did go on, but in a staggered way, with partial conversations adding up slowly into a bigger picture of progress. What stopped this happening successfully was perhaps our own fear of the capacity development facilitation process taking undue prominence within the programme, rivalling management and strategic processes in ways that would not have been welcomed by those with formal leadership roles. Do facilitators and learners within capacity development therefore need to claim more leadership space and be stronger advocates for their insights into strategic direction and management decisions affecting the higher level goals of programmes of which they are a part? Conversely, should programme leaders take 
more control of the facilitation of capacity development as a critical success factor?

Thinking about capacity development, it is perhaps too easy to lose sight of the fact that capacity is built through acts of learning. As facilitators, we can support capacity development processes and activities but unless people feel genuinely motivated to learn, participation in them is not going to lead to new capabilities. Undermotivated participation results in people increasing their understanding but not their knowledge (like understanding how to ride a bicycle in principle, but not knowing what it is like because of never having tried it out). So in Africa Adapt, we should have tried harder to keep in view people's motivations for learning. These could have been better understood by the knowledge sharing capacity development advisers finding out what questions KSOs were motivated to ask, and what the value of learning the answers to these questions would be to them in their organisational context and profession. These factors were touched on in an emergent way within responsive mentoring discussions, but the extent to which they received attention was dependent on KSOs presenting issues around motivation for and the value of learning. As knowledge sharing capacity development advisers, if we had a better understanding of these questions and the value of answers to them, we could have tailored the approach.

In taking a systemic approach to facilitating capacity development, we implicitly defined a boundary for the system whose capacity we wanted to develop, but perhaps not consciously enough. Establishing a systems boundary is important because it allows the design and planning of the capacity development to focus not spreading resources too thinly or targeting them in the wrong direction. With Africa Adapt, we implicitly took our system boundary to be the consortium of project partners. If we had more consciously explored this demarcation, we would probably have more clearly understood how bounding the system in this way actively structured the kinds of relationships Africa Adapt could have with other capacity development facilitation initiatives in the area of knowledge networking. So the risk of taking a systematic approach and only implicitly setting the boundary is to end up seeing the Africa Adapt system in too isolated a way from others that impact upon and will be affected by the facilitation of capacity development in our system. Perhaps a more conscious approach could be found that did not overwhelm us with the need to fully understand and relate to all related and nested systems. In future, we could try to be conscious of the system boundary as being provisionally drawn and implying the need for the facilitation team and project leadership to scan the horizon for changes in nearby systems and adjust the boundary in light of new developments.

\section{Conclusion}

In seeking to take a more systemic approach to the facilitation of capacity development in the Africa Adapt project, we have learnt that it may be particularly appropriate when applied to contexts that have complicated features (spatial, linguistic, sectoral, methodological, etc.). A complicated context tends to enable a systemic approach and vice versa. We have also found that context, in the way it shapes opportunities to apply new capabilities, may differentially favour learners according to their existing mindsets and attitudes.

We have also learnt that our practice needs to be more aware of a couple of potential pitfalls from transferring Morgan's (2006) theory too literally or unsubtly. It appears to be quite easy to end up facilitating capacity development in a way that is comprehensive but without being really systemic. So, for example, our practice supported a mix of hard and soft skills, used different methods and sequencing and sought to base this on different sources of evidence about capability needs and opportunities. This comprehensive engagement perhaps failed to be systemic because it was based on an initial understanding of the system whose capacity was to be developed, but this understanding was not regularly refreshed and became a snapshot that was eventually out of date. This is particularly poignant when we consider that one of the major new factors influencing the system was our facilitation of capacity development itself. So this points to the need for a systemic approach to capacity development, to be both dynamically updated and reflexive in its appraisal of the capability challenge.

The next pitfall is to focus too much on the system as a whole, while failing to pay sufficient 
attention to the micro-relationships between components within that system. In our case, this was exemplified by our attention to capability at the level of the partner organisations in terms of their ability to support the KSOs, but missing the changing dynamic within the relationships of the KSOs to their line managers and KSOs' personal learning motivations. Perhaps this calls out the need to retain a focus on the different parts of the system as well as cultivating an awareness of the whole that these parts make up.

More broadly, in seeking to facilitate capacity development, we have learnt that our practice needs to take more account of power. Power relates to the application of methods, and to the management of the wider intervention of which the capacity development process is a part. In relation to the application of methods, power is a significant modulating force influencing the context within which a method is applied. For instance, our application of mentoring methods sought to ensure that power in terms of line management relations was bracketed out by ensuring that the mentors were not part of the management structure of Africa Adapt. However, this was not the only power relationship that could potentially modulate mentoring, and the wider positional power of facilitators needs to be

\section{Notes}

* I would like to thank the Africa Adapt partners, IDRC, DFID and particularly Africa Adapt's Knowledge Sharing Officers for the inspiration and opportunity to write this article.

1 Africa Adapt is collaboratively hosted by four organisations: Environment and Development in the Third World (ENDA-TM), the Forum for Agricultural Research in Africa (FARA), IGAD Climate Prediction and Applications Centre

\section{References}

Africa Adapt (2009) About Africa Adapt, www.africa-adapt.net/AA/AboutUs.aspx (accessed 20 January 2010)

IDRC (2007) CCAA Program Activities, www.idrc.ca/ccaa/ev-94557-201-1-DO TOPIC.html (accessed 20 January 2010) taken into account. This perhaps suggests the need to consider dimensions of power beyond the formal to include, for example, informal and hidden power.

Turning to power as it relates to the management of the wider intervention of which the capacity development process is a part, the facilitators' agency (or lack of it) is evident. For example, the facilitators did not participate in the management space within Africa Adapt, and therefore it was less easy for management decisions to be fully informed by insights formed through the capacity development process. Interestingly however, the implication that facilitators of capacity development should be agents within management is in tension with the conclusion above that the more closely associated with management power facilitators become, the less able they are to sustain an enabling context for capacity development.

Perhaps in combination, these twin reflections on power suggest that the ideal of facilitating capacity development from a position of neutrality is a fiction and that what is actually more appropriate is to make the positional power of facilitators more transparent and a subject for negotiation, reflection and accountability.

(ICPAC), and the Institute of Development Studies (IDS) - www.africa-adapt.net (accessed 27 March 2010).

2 The network is jointly funded by Canada's International Development Research Centre (IDRC) and the UK Department for International Development (DFID) Climate Change Adaptation in Africa Programme www.idrc.ca/ccaa/ (accessed 27 March 2010).

Morgan, P. (2006) The Concept of Capacity, Maastricht: European Centre for Development Policy Management (ECDPM)

Taylor, P. and Clarke, P. (2008) Capacity for a Change, Brighton: IDS 